\title{
Vitamin D Supplementation Regulates Postoperative Serum Levels of PD-L1 in Patients with Digestive Tract Cancer and Improves Survivals in the Highest Quintile of PD-L1: A Post Hoc Analysis of the AMATERASU Randomized Controlled Trial
}

\author{
Makoto Morita ${ }^{1,2}{ }^{2}$, Mai Okuyama ${ }^{1}$, Taisuke Akutsu ${ }^{1}$, Hironori Ohdaira ${ }^{3}$, Yutaka Suzuki ${ }^{3}$ and $^{1}$ \\ Mitsuyoshi Urashima $1, *$ (D)
}

1 Division of Molecular Epidemiology, The Jikei University School of Medicine, 3-25-8 Nishi-Shimbashi, Minato-Ku, Tokyo 105-8461, Japan; external.mm@gmail.com (M.M.); maiokuyama0511@gmail.com (M.O.); taisuke0107.jusom@gmail.com (T.A.)

2 Pfizer Japan Inc., Shinjuku Culture Quint Bldg, 3-22-7 Yoyogi, Shibuya-ku, Tokyo 151-8589, Japan

3 Department of Surgery, International University of Health and Welfare Hospital, 537-3 Iguchi, Nasushiobara, Tochigi 329-2763, Japan; ohdaira@iuhw.ac.jp (H.O.); yutaka@iuhw.ac.jp (Y.S.)

* Correspondence: urashima@jikei.ac.jp; Tel.: +81-3-3433-1111 (ext. 2405)

\section{check for}

updates

Citation: Morita, M.; Okuyama, M.; Akutsu, T.; Ohdaira, H.; Suzuki, Y.;

Urashima, M. Vitamin D

Supplementation Regulates

Postoperative Serum Levels of PD-L1

in Patients with Digestive Tract

Cancer and Improves Survivals in the

Highest Quintile of PD-L1: A Post

Hoc Analysis of the AMATERASU

Randomized Controlled Trial.

Nutrients 2021, 13, 1987.

https://doi.org/10.3390/nu13061987

Received: 9 May 2021

Accepted: 5 June 2021

Published: 9 June 2021

Publisher's Note: MDPI stays neutral with regard to jurisdictional claims in published maps and institutional affiliations.

Copyright: (c) 2021 by the authors. Licensee MDPI, Basel, Switzerland. This article is an open access article distributed under the terms and conditions of the Creative Commons Attribution (CC BY) license (https:// creativecommons.org/licenses/by/ $4.0 /)$.

\begin{abstract}
Because vitamin D responsive elements have been found to be located in the PD-L1 gene, vitamin D supplementation was hypothesized to regulate serum PD-L1 levels and thus alter survival time of cancer patients. A post hoc analysis of the AMATERASU randomized, double-blind, placebocontrolled trial of postoperative vitamin D3 supplementation (2000 IU/day) in 417 patients with stage I to stage III digestive tract cancer from the esophagus to the rectum was conducted. Postoperative serum PD-L1 levels were measured by ELISA and divided into quintiles (Q1-Q5). Serum samples were available for 396 (95.0\%) of the original trial. Vitamin D supplementation significantly $(p=0.0008)$ up-regulated serum PD-L1 levels in the lowest quintile (Q1), whereas it significantly $(p=0.0001)$ down-regulated them in the highest quintile (Q5), and it did not either up- or down-regulate them in the middle quintiles (Q2-Q4). Significant effects of vitamin D supplementation, compared with placebo on death (HR, 0.34; 95\% CI, 0.12-0.92) and relapse/death (HR, 0.37; 95\% CI, 0.15-0.89) were observed in the highest quintile (Q5) of serum PD-L1, whereas significant effects were not observed in other quintiles $\left(P_{\text {interaction }}=0.02\right.$ for death, $P_{\text {interaction }}=0.04$ for relapse $/$ death $)$. Vitamin $\mathrm{D}$ supplementation significantly reduced the risk of relapse/death to approximately one-third in the highest quintile of serum PD-L1.
\end{abstract}

Keywords: vitamin D; supplement; PD-L1; cancer; serum; soluble; survival; randomized; placebo

\section{Introduction}

Programmed death-ligand 1 (PD-L1) is expressed on a part of cancer cells to suppress anti-cancer immunity by interacting with the programmed death-1 (PD-1) receptor expressed on immune cells [1]. Indeed, blocking this interaction by administering monoclonal antibodies targeting either the PD-1 or the PD-L1 molecule improves the prognosis of patients with cancer, at least in part [2]. Moreover, PD-L1 is constitutively expressed at low levels on non-cancer cells, e.g., antigen-presenting cells, vascular endothelial cells, and pancreatic islet cells, which may induce immune tolerance by maintaining the quiescence of autoreactive immune cells [1].

Membrane-bound forms of PD-L1 are also expressed on the surface of exosomes, whereas soluble forms of PD-L1 are generated as splice variants or by proteolytic cleavage of membrane-bound forms, and both are secreted into the extracellular space and blood 
stream [3-5]. Thus, total levels of serum PD-L1 measured by enzyme-linked immunosorbent assay (ELISA) may reflect the sum of both exosomal and soluble forms of PD-L1. Serum PD-L1 was considered to be functional and shown in vitro to induce apoptosis of CD4+ and CD8+ T cells derived from either a patient with cancer or a healthy person $[6,7]$. In addition, we and our colleagues reported that serum PD-L1 levels were increased up to seven-fold in pregnant women compared with age-matched non-pregnant women, and further demonstrated in vitro that the increased serum PD-L1 of pregnant women suppressed both autogenic and allogeneic immune reactions, as well as cytokine production of immune cells [8]. In fact, a recent meta-analysis including a total of 21 studies demonstrated that elevated serum PD-L1 levels were associated with worse survival of patients with cancer [9]. In particular, higher postoperative, but not preoperative, plasma total PD-L1, in addition to exosomal PD-L1, was shown to be associated with poor survival in patients with gastric cancer [10]. Thus, not only relying on immune checkpoint inhibitors, but also reducing serum PD-L1 levels after operation, is another distinct strategy to improve the prognosis of patients with cancer. However, few strategies are suitable for clinical use at the moment except for therapeutic plasma exchange [11].

Vitamin D is a precursor of $1,25(\mathrm{OH}) \mathrm{D}$, which is a potent steroid hormone, and has been reported to have both positive and negative transcriptional regulations of gene expressions relating to innate immune responses through the vitamin D receptor in the target cell [12]. Of interest, vitamin D-responsive elements have been found to be located in an intronic region of the PD-L1 gene [13]. However, there are few reports of the interaction between serum PD-L1 and vitamin D. Vitamin D supplementation was hypothesized to regulate the serum levels of PD-L1 and thus change survival time of patients with cancer. We and our colleagues previously conducted the AMATERASU randomized, double-blind, placebo-controlled trial of postoperative vitamin D3 supplementation (2000 IU/day) in 417 patients with stage I to stage III digestive tract cancer from the oesophagus to the rectum who underwent curative surgery [14]. By conducting a post hoc analysis of the AMATERASU trial, the aim of this study was thus to examine the effects of vitamin D supplementation on the serum PD-L1 levels 1 year after starting supplements and on survival in each quintile of serum PD-L1 levels in patients with digestive tract cancer.

\section{Materials and Methods}

\subsection{Trial Design}

This study was a post hoc analysis of the AMATERASU trial (UMIN000001977) conducted in Japan, the details of which have been previously reported [14]. Briefly, 417 patients with digestive tract cancers from the oesophagus to the rectum participated in a randomized, double-blind, placebo-controlled trial to compare the effects of vitamin D3 supplements (2000 IU/day) and placebo on relapse and/or death at an allocation ratio of 3:2 at the International University of Health and Welfare Hospital (Otawara, Tochigi, Japan) between January 2010 and February 2018. The trial protocol was approved by the ethics committee of the International University of Health and Welfare Hospital (Otawara, Tochigi, Japan) (ethics approval code: 13-B-263), as well as the Jikei University School of Medicine (Nishi-shimbashi, Tokyo, Japan) (ethics approval code: 21-216 (6094)). Written, informed consent was obtained from each participating patient before surgery.

\subsection{Participants}

Details of the inclusion and exclusion criteria were described in the original report [14]. Briefly, the trial included patients not taking vitamin D supplements with stage I to stage III digestive tract cancers (esophageal, gastric, small intestinal, and colorectal) who underwent curative surgery with complete tumor resection. The outcome of relapse or death was confirmed by regular outpatient follow-up. The elapsed time to relapse or death was calculated from the time of randomization (i.e., time from starting the study supplements). 


\subsection{Measurement of Serum PD-L1 Levels}

Serum samples for PD-L1 measurements were collected after the surgery (23 days, interquartile range (IQR): $13-43.5$ days) and just before the start of vitamin D/placebo supplementation. The serum PD-L1 level was also measured 1 year after starting vitamin $\mathrm{D} /$ placebo supplements. The serum samples were stored at $-80^{\circ} \mathrm{C}$ prior to use. Serum PDL1 levels were measured by a member of the research team, who was blind to randomized groups and clinical information including outcomes, which were fixed prior to statistical analyses, using ELISA kits from Abcam (\#ab214565) (Cambridge, MA, USA), according to the manufacturer's protocols. The lower detection limit for serum PD-L1 of the ELISA kit was $3.9 \mathrm{pg} / \mathrm{mL}$, and the upper detection limit was $1300 \mathrm{pg} / \mathrm{mL}$.

\subsection{Evaluation of Other Covariates}

The details of the analysis of histopathological subtypes [15], analysis of p53 protein, vitamin D receptor (VDR), Ki-67 by immunohistochemistry [16], and serum levels of bioavailable 25-hydroxyvitamin $\mathrm{D}(25(\mathrm{OH}) \mathrm{D})$ [17] have been described in previous reports. Histopathological subtypes were not mutually exclusive because there could be multiple subtypes; p53-positive was defined as a positive nuclear percentage in the tumor epithelium greater than $10 \%$. VDR was defined as a score using a semiquantitative scoring system, and $\mathrm{Ki}-67$ was defined as the positive nuclear staining percentage in tumor epithelium. Bioavailable $25(\mathrm{OH}) \mathrm{D}$ was calculated using serum concentrations of $25(\mathrm{OH}) \mathrm{D}$, vitamin $\mathrm{D}$ binding protein, albumin, and single-nucleotide polymorphisms of vitamin $\mathrm{D}$ binding protein.

\subsection{Statistical Analysis}

All patients who underwent randomization and for whom residual serum samples were available were included in this analysis. Spearman's rank correlation coefficient (RHO) was used to quantify the strengths of associations between two continuous variables: RHO $\geq 0.4$, strong; $0.4>$ RHO $\geq 0.2$, moderate; and RHO $<0.2$, weak. Non-parametric continuous variables and dichotomous variables were compared between groups by the Mann-Whitney test and the chi-squared test, respectively. Changes in serum PD-L1 levels from baseline to 1 year later in either the vitamin $\mathrm{D}$ or placebo group were analyzed using the Wilcoxon signed-rank test.

Relapse and death-related outcomes were assessed according to the randomization group by whether or not supplements were taken. The effects of vitamin D and placebo on the risks of outcomes, i.e., total death and relapse/death, were estimated using NelsonAalen cumulative hazard curves. A Cox proportional hazards model was used to determine hazard ratios (HRs) and $95 \%$ confidence intervals (CIs) for the outcomes. To evaluate the effects of vitamin D supplementation on relapse, cumulative incidence functions were applied by considering patient deaths due to causes other than cancer relapse as a competing risk; competing risk regression was performed using subdistribution hazard ratios (SHRs) and 95\% CIs [18]. When the 95\% CI did not include 1, the HR and SHR were considered significant. To clarify whether vitamin D supplementation differed significantly among quintiles of serum PD-L1 levels (Q1-Q5), the $p$ for interaction was analyzed on the basis of a Cox regression model including three variables (vitamin $\mathrm{D}$ group, the highest quintile of serum PD-L1 (Q5), and both the vitamin D group and the highest quintile (Q5) of serum PD-L1) by two-way interaction tests comparing the subgroup of the highest quintile of serum PD-L1 and the others. Values of $p$ for interaction with two-sided $p<0.05$ were considered significant. All data were analyzed using Stata 14.0 (StataCorp LP; College Station, TX, USA).

\section{Results}

\subsection{Study Population}

Of the 417 patients with digestive tract cancers who were randomly assigned to receive vitamin D supplements $(n=251,60 \%)$ or placebo $(n=166,40 \%)$, ELISA results for serum 
PD-L1 were available for 396 (95.0\%) of the original AMATERASU trial participants (244 $(97.2 \%)$ of the vitamin D group and $152(91.6 \%)$ of the placebo group) because they were not sampled from patients or used up for other studies (Figure 1). However, 1 year after starting supplements, the number of available serum PD-L1 samples was further reduced to $319(80.6 \%)(198(81.1 \%)$ of the vitamin D group and 121 (79.6\%) of the placebo group), due to death, transfer to other hospitals, sampled not being taken from patients, or samples being used up for other studies. The median follow-up of these 396 patients was 3.5 years (interquartile range (IQR): $2.4-5.4$ years).

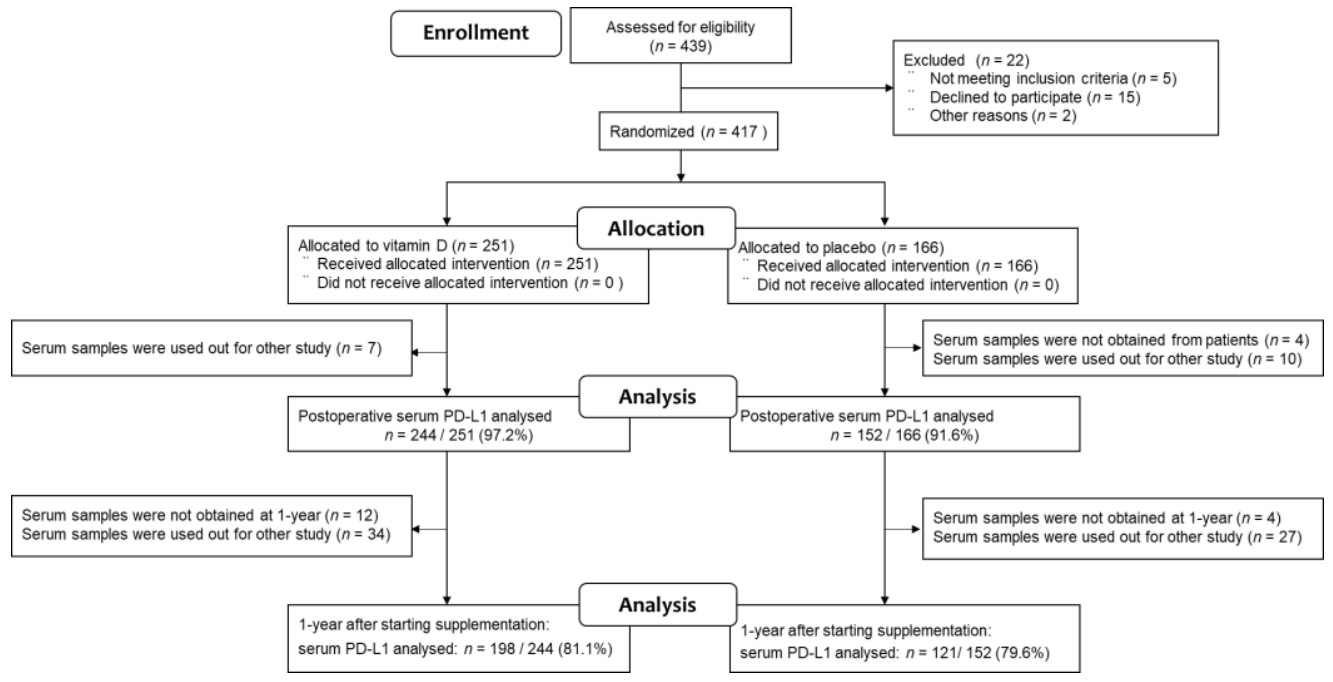

Figure 1. Patient flowchart through the present post hoc analysis.

\subsection{Patients' Characteristics Stratified by Vitamin D Group and Placebo Group}

Patients' characteristics by vitamin D group and placebo group are shown in Table 1. The 3:2 ratio of assignment to the vitamin $\mathrm{D}$ and placebo groups was generally maintained for all variables. Of the 396 participants, $33 \%$ were women. The median age (IQR) was $66(60-74)$ years, and the median body mass index was $21.9(19.8-23.8) \mathrm{kg} / \mathrm{m}^{2}$. Percentages of cancer sites were as follows: esophageal, $9 \%$; gastric, $42 \%$; small intestinal, $1 \%$; and colorectal, $48 \%$. Disease stages were I, II, and III in $44 \%, 26 \%$, and $30 \%$ of patients, respectively.

Table 1. Patients' characteristics stratified by Vitamin D vs. Placebo.

\begin{tabular}{|c|c|c|}
\hline$n=396$ & Vitamin D $n=244$ & Placebo $n=152$ \\
\hline $25(\mathrm{OH}) \mathrm{D}, \mathrm{ng} / \mathrm{mL}$ & $n=241$ & $n=152$ \\
\hline median & 21 & 21 \\
\hline $\mathrm{IQR}^{\mathrm{a}}(25-75 \%)$ & $(17-27)$ & $(14.5-26)$ \\
\hline $\begin{array}{l}25(\mathrm{OH}) \mathrm{D}, \mathrm{ng} / \mathrm{mL} 1 \text { year after } \\
\text { supplementation }\end{array}$ & $n=208$ & $n=132$ \\
\hline median & 41 & 21 \\
\hline $\mathrm{IQR}^{\mathrm{a}}(25-75 \%)$ & $(33-55)$ & $(15-27)$ \\
\hline Bioavailable 25(OH)D, ng/mL & $n=214$ & $n=136$ \\
\hline median & 1.8 & 1.6 \\
\hline $\mathrm{IQR}^{\mathrm{a}}(25-75 \%)$ & $(1.2-2.8)$ & $(1.1-2.3)$ \\
\hline $\begin{array}{l}\text { Bioavailable } 25(\mathrm{OH}) \mathrm{D}, \mathrm{ng} / \mathrm{mL} 1 \text { year } \\
\text { after supplementation }\end{array}$ & $n=177$ & $n=117$ \\
\hline
\end{tabular}


Table 1. Cont.

\begin{tabular}{|c|c|c|}
\hline$n=396$ & Vitamin D $n=244$ & Placebo $n=152$ \\
\hline median & 5.0 & 2.2 \\
\hline $\mathrm{IQR}^{\mathrm{a}}(25-75 \%)$ & $(3.4-7.5)$ & $(1.6-3.3)$ \\
\hline Sex, $n(\%)$ & $n=244$ & $n=152$ \\
\hline Male & $171(70)$ & $94(62)$ \\
\hline Female & $73(30)$ & $58(38)$ \\
\hline Age, $y$ & $n=244$ & $n=152$ \\
\hline median & 67 & 64 \\
\hline $\mathrm{IQR}^{\mathrm{a}}(25-75 \%)$ & $(61-75)$ & $(58-70)$ \\
\hline Body mass index $\left(\mathrm{kg} / \mathrm{m}^{2}\right)$ & $n=242$ & $n=151$ \\
\hline median & 21.9 & 22.1 \\
\hline $\mathrm{IQR}^{\mathrm{a}}(25-75 \%)$ & $(19.8-24.0)$ & $(20.0-23.7)$ \\
\hline History of other cancers, $n(\%)$ & $8(3)$ & $7(5)$ \\
\hline Comorbid condition, $n(\%)$ & $n=244$ & $n=152$ \\
\hline Hypertension & $101(41)$ & $54(36)$ \\
\hline Diabetes Mellitus & $44(18)$ & $21(14)$ \\
\hline Endocrine Disease & $32(13)$ & $16(11)$ \\
\hline Coronary Artery Disease & $16(7)$ & $2(1)$ \\
\hline Stroke & $10(4)$ & $6(4)$ \\
\hline Chronic Kidney Disease & $4(2)$ & $1(0.7)$ \\
\hline Asthma & $3(1)$ & $0(0)$ \\
\hline Orthopaedic disease & $1(0.4)$ & $1(0.7)$ \\
\hline Site of cancer, $n(\%)$ & $n=244$ & $n=152$ \\
\hline Oesophagus & $22(9)$ & $15(10)$ \\
\hline Stomach & $104(43)$ & $64(42)$ \\
\hline Small bowel & $1(0.4)$ & $1(0.7)$ \\
\hline Colorectal & $117(48)$ & $72(47)$ \\
\hline Stage, $n(\%)$ & $n=244$ & $n=152$ \\
\hline I & $113(46)$ & $61(40)$ \\
\hline II & $61(25)$ & $43(28)$ \\
\hline III & $70(29)$ & $48(32)$ \\
\hline \multicolumn{3}{|l|}{ Pathology $b$} \\
\hline Adenocarcinoma, $n(\%)$ & $n=244$ & $n=152$ \\
\hline Well-differentiated & $137(56)$ & $75(49)$ \\
\hline Moderately differentiated & $93(38)$ & $66(43)$ \\
\hline Poorly differentiated & $43(18)$ & $32(21)$ \\
\hline Signet ring cell & $18(7)$ & $22(14)$ \\
\hline Mucinous & $18(7)$ & $8(5)$ \\
\hline Papillary & $11(5)$ & $4(3)$ \\
\hline Squamous cell carcinoma, $n(\%)$ & $20(8)$ & $11(7)$ \\
\hline P53 expression, $n(\%)$ & $n=214$ & $n=140$ \\
\hline
\end{tabular}


Table 1. Cont.

\begin{tabular}{ccc}
\hline$n=396$ & Vitamin D $n=\mathbf{2 4 4}$ & Placebo $n=\mathbf{1 5 2}$ \\
\hline None & $35(16)$ & $28(20)$ \\
\hline Faintly expressed: $>0 \% \&<10 \%$ & $43(20)$ & $31(22)$ \\
\hline Strongly expressed: $\geq 10 \% \&<50 \%$ & $30(14)$ & $26(19)$ \\
\hline Overexpressed: $\geq 50 \%$ & $106(50)$ & $55(39)$ \\
\hline Vitamin D receptor expression, $n(\%)$ & $n=214$ & $n=140$ \\
\hline Quartile 1, & $59(28)$ & $33(24)$ \\
\hline Quartile 2, & $54(25)$ & $35(25)$ \\
\hline Quartile 3, & $51(24)$ & $37(25)$ \\
\hline Quartile 4, & $50(23)$ & $n=140$ \\
\hline Ki67 expression, $n(\%)$ & $n=214$ & $25(18)$ \\
\hline Quartile 1, & $37(17)$ & $40(29)$ \\
\hline Quartile 2, & $76(36)$ & $29(21)$ \\
\hline Quartile 3, & $30(14)$ & $46(33)$ \\
\hline Quartile 4, & $71(33)$ & $56(37)$ \\
\hline Adjuvant chemotherapy, $n(\%)$ & $84(34)$ & \\
\hline
\end{tabular}

${ }^{\mathrm{a}} \mathrm{IQR}=$ Interquartile range. ${ }^{\mathrm{b}}$ Because many patients had multiple histopathologic components, histopathologic subgroups were not mutually exclusive from each other.

\subsection{Serum PD-L1 Levels before and after Starting Supplements}

Serum PD-L1 levels were assessed in 396 patients (Figure 2A). The median (IQR) level was $55.5(44.2-70.2) \mathrm{pg} / \mathrm{mL}$, with the distribution skewed to the right. Strong associations between serum PD-L1 levels before and after starting supplements were observed in the total sample (Figure $2 \mathrm{~B}$ ), in the vitamin D group (Figure $2 \mathrm{C}$ ), and in the placebo group (Figure 2D).
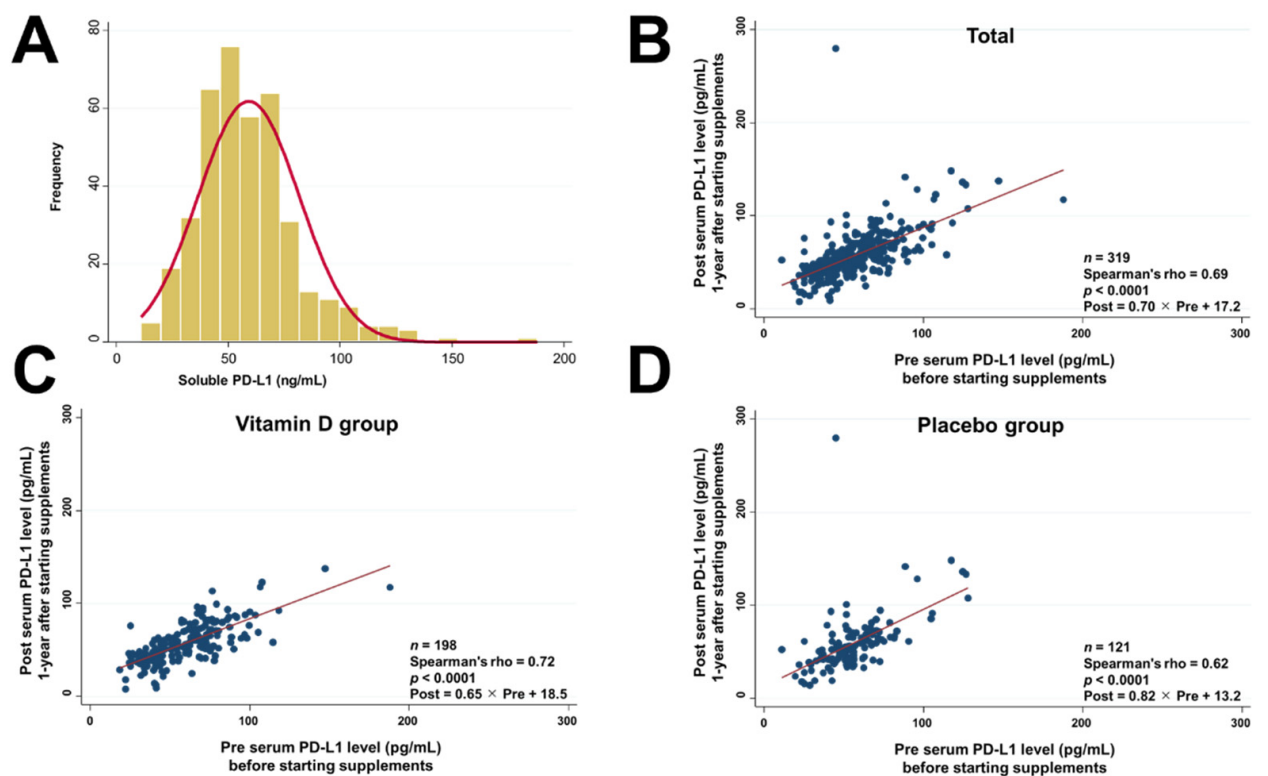

Figure 2. Histogram of serum PD-L1 levels (A). Scatter plot between pre serum PD-L1 and post serum PD-L1 levels in all patients (B), in the vitamin D group (C), and in the placebo group (D). Spearman's rank correlation coefficient (RHO) was used to quantify the strength of the association. The equation was calculated by linear regression analysis. 


\subsection{Patients' Characteristics Stratified by Quintiles of Serum PD-L1 Levels}

Patients' characteristics in subgroups stratified by quintiles of serum PD-L1 levels are shown in Table 2. There were no differences in serum 25(OH)D levels or bioavailable $25(\mathrm{OH}) \mathrm{D}$ levels before vitamin $\mathrm{D}$ intervention among subgroups. Moreover, distributions of sex, body mass index, history of other cancers, comorbid conditions (except that a previous history of coronary artery disease was more frequent in Q5 than in other quintiles), site of cancers, stage, pathology, p53 expression, VDR expression, and adjuvant chemotherapy were also not different. There was only a difference for age, which was significantly higher in higher quintiles.

Table 2. Patients' characteristics in subgroups stratified by quintiles of serum PD-L1 levels.

\begin{tabular}{|c|c|c|c|c|c|c|}
\hline & Total $n=396$ & Q1 $n=79$ & Q2 $n=80$ & Q3 $n=79$ & Q4 $n=79$ & Q5 $n=79$ \\
\hline $\begin{array}{c}\text { Median }\left(\mathrm{IQR}^{\mathrm{b}}\right), \\
\mathrm{pg} / \mathrm{mL}\end{array}$ & $55.5(44.2-70.2)$ & $34.4(26.1-38.7)$ & $45.7(44.2-49.1)$ & $55.5(53.4-58.7)$ & $67.3(64.7-70.2)$ & $\begin{array}{c}86.8 \\
(78.8-103.6)\end{array}$ \\
\hline \multicolumn{7}{|c|}{ Intervention } \\
\hline$\underset{(\%)}{\operatorname{Vitamin}} \mathrm{D}, n$ & $244(62)$ & $54(68)$ & $41(51)$ & $44(56)$ & $48(61)$ & $57(72)$ \\
\hline Placebo, $n(\%)$ & $152(38)$ & $25(32)$ & $39(49)$ & $35(44)$ & $31(39)$ & $22(28)$ \\
\hline \multicolumn{7}{|c|}{$25(\mathrm{OH}) \mathrm{D}^{\mathrm{c}}, \mathrm{ng} / \mathrm{mL}$ median $\left(\mathrm{IQR}^{\mathrm{b}}\right)$} \\
\hline All & $21(16-27)$ & $22(17-28)$ & $20(17-27)$ & $20(14-25)$ & $22(17-28)$ & $20(14-26)$ \\
\hline $\begin{array}{l}\text { Vitamin D sup- } \\
\text { plementation }\end{array}$ & $21(17-27)$ & $23(18-28)$ & $22(18-28)$ & $19(15-25)$ & $23(19-30)$ & $20(14-26)$ \\
\hline $\begin{array}{l}\text { Placebo supple- } \\
\text { mentation }\end{array}$ & $21(15-26)$ & $21(16-25)$ & $20(15-27)$ & $22(13-26)$ & $21(14-28)$ & $19(15-26)$ \\
\hline \multicolumn{7}{|c|}{$25(\mathrm{OH}) \mathrm{D}^{\mathrm{c}}, \mathrm{ng} / \mathrm{mL} 1$ year after supplementation median $\left(\mathrm{IQR}^{\mathrm{b}}\right)$} \\
\hline All & $33(21-47)$ & $32(20-47)$ & $32(19-42)$ & $32(21-41)$ & $31(22-47)$ & $37(21-55)$ \\
\hline $\begin{array}{l}\text { Vitamin D sup- } \\
\text { plementation. }\end{array}$ & $41(33-55)$ & $40(30-54)$ & $40(35-54)$ & $40(33-54)$ & $35(45-58)$ & $44(35-60)$ \\
\hline $\begin{array}{l}\text { Placebo supple- } \\
\text { mentation }\end{array}$ & $21(15-27)$ & $23(17-30)$ & $19(13-28)$ & $22(16-29)$ & $22(18-25)$ & $16(11-23)$ \\
\hline \multicolumn{7}{|c|}{ Bioavailable $25(\mathrm{OH}) \mathrm{D}^{\mathrm{c}}, \mathrm{ng} / \mathrm{mL}$ median $\left(\mathrm{IQR}^{\mathrm{b}}\right)$} \\
\hline All & $1.71(1.18-2.59)$ & $1.73(1.33-2.73)$ & $1.79(1.28-2.62)$ & $1.75(1.04-2.31)$ & $1.94(1.26-2.87)$ & $1.45(1.00-2.16)$ \\
\hline $\begin{array}{l}\text { Vitamin D sup- } \\
\text { plementation }\end{array}$ & $1.80(1.23-2.79)$ & $1.97(1.17-3.06)$ & $1.95(1.54-2.74)$ & $1.70(1.14-2.22)$ & $2.17(1.57-3.12)$ & $1.52(1.02-2.48)$ \\
\hline $\begin{array}{c}\text { Placebo supple- } \\
\text { mentation }\end{array}$ & $1.63(1.08-2.28)$ & $1.68(1.53-2.02)$ & $1.71(1.25-2.42)$ & $1.90(0.97-2.62)$ & $1.47(0.97-2.40)$ & $1.26(0.97-1.65)$ \\
\hline \multicolumn{7}{|c|}{ Bioavailable $25(\mathrm{OH}) \mathrm{D}^{\mathrm{c}}, \mathrm{ng} / \mathrm{mL} 1$ year after supplementation median (IQR ${ }^{\mathrm{b}}$ ) } \\
\hline All & $3.62(2.16-6.20)$ & $3.59(2.43-5.05)$ & $3.48(2.00-6.25)$ & $3.35(1.82-5.71)$ & $3.93(2.07-6.94)$ & $4.20(2.16-6.88)$ \\
\hline $\begin{array}{l}\text { Vitamin D sup- } \\
\text { plementation. }\end{array}$ & $5.05(3.37-7.51)$ & $4.31(3.25-7.13)$ & $4.85(3.60-7.58)$ & $5.21(3.28-6.96)$ & $5.58(3.43-8.36)$ & $6.19(2.82-7.54)$ \\
\hline $\begin{array}{l}\text { Placebo supple- } \\
\text { mentation }\end{array}$ & $2.25(1.60-3.32)$ & $2.43(1.76-3.64)$ & $2.21(1.27-3.31)$ & $2.22(1.66-3.74)$ & $2.84(1.64-3.55)$ & $2.16(1.41-2.53)$ \\
\hline \multicolumn{7}{|c|}{ Sex, $n(\%)$} \\
\hline Male & $265(67)$ & $48(61)$ & $47(59)$ & $58(73)$ & $50(63)$ & $62(78)$ \\
\hline Female & $131(33)$ & $31(39)$ & $33(41)$ & $21(27)$ & $29(37)$ & $17(22)$ \\
\hline $\begin{array}{c}\text { Age, } y \\
\text { median }\left(\mathrm{IQR}^{\mathrm{b}} \text { ) }\right.\end{array}$ & $66(60-74)$ & $63(57-70)$ & $64(59-73)$ & $64(57-70)$ & $70(62-75)$ & $72(64-78)$ \\
\hline \multicolumn{7}{|c|}{ Body mass index $\left(\mathrm{kg} / \mathrm{m}^{2}\right)^{\mathrm{d}}$} \\
\hline median (IQR b) & $21.9(19.8-23.8)$ & $22.4(20.0-24.0)$ & $21.4(20.0-23.5)$ & $21.7(20.4-24.2)$ & $21.9(20.0-23.7)$ & $21.6(19.2-24.1)$ \\
\hline $\begin{array}{l}\text { History of other } \\
\text { cancers, } n(\%)\end{array}$ & $15(3.8)$ & $4(5.1)$ & $3(3.8)$ & $5(6.3)$ & $2(2.5)$ & $1(1.3)$ \\
\hline
\end{tabular}


Table 2. Cont.

\begin{tabular}{|c|c|c|c|c|c|c|}
\hline & Total $n=396$ & Q1 $n=79$ & Q2 $n=80$ & Q3 $n=79$ & Q4 $n=79$ & Q5 $n=79$ \\
\hline \multicolumn{7}{|c|}{ Comorbid condition, $n(\%)^{\mathrm{a}}$} \\
\hline Hypertension & $155(39)$ & $25(32)$ & $25(32)$ & $41(52)$ & $28(35)$ & $36(46)$ \\
\hline $\begin{array}{l}\text { Diabetes } \\
\text { Mellitus }\end{array}$ & $65(16)$ & $11(14)$ & $11(14)$ & $13(16)$ & $12(15)$ & $18(23)$ \\
\hline $\begin{array}{l}\text { Endocrine } \\
\text { Disease }\end{array}$ & $48(12)$ & $9(11)$ & $13(16)$ & $11(14)$ & $7(8.9)$ & $8(10)$ \\
\hline $\begin{array}{c}\text { Coronary } \\
\text { Artery Disease }\end{array}$ & $18(4.5)$ & $2(2.5)$ & $3(3.8)$ & $3(3.8)$ & $2(2.5)$ & $8(10.1)$ \\
\hline Stroke & $16(4.0)$ & $1(1.3)$ & $2(2.5)$ & $4(5.1)$ & $3(3.8)$ & $6(7.6)$ \\
\hline $\begin{array}{l}\text { Chronic Kidney } \\
\text { Disease }\end{array}$ & $5(1.3)$ & $0(0.0)$ & $0(0.0)$ & $0(0.0)$ & 2. (2.5) & $3(3.8)$ \\
\hline Asthma & $3(0.8)$ & $0(0.0)$ & $0(0.0)$ & $0(0.0)$ & $1(1.3)$ & $2(2.5)$ \\
\hline $\begin{array}{l}\text { Orthopaedic } \\
\text { disease }\end{array}$ & $2(0.5)$ & $0(0.0)$ & $1(1.3)$ & $1(1.3)$ & $0(0.0)$ & $0(0.0)$ \\
\hline \multicolumn{7}{|c|}{ Site of cancer, $n(\%)^{\mathrm{a}}$} \\
\hline Oesophagus & $37(9.3)$ & $3(3.8)$ & $6(7.5)$ & $7(8.9)$ & $11(13.9)$ & $10(12.7)$ \\
\hline Stomach & $168(42.4)$ & $39(49.4)$ & $37(46.3)$ & $29(36.7)$ & $28(35.4)$ & $35(44.3)$ \\
\hline Small bowel & $2(0.5)$ & $0(0.0)$ & $0(0.0)$ & $1(1.3)$ & $1(1.3)$ & $0(0.0)$ \\
\hline Colorectal & $189(47.7)$ & $37(46.8)$ & $37(46.3)$ & $42(53.2)$ & $39(49.4)$ & $34(43.0)$ \\
\hline \multicolumn{7}{|c|}{ Stage, $n(\%)^{\mathrm{a}}$} \\
\hline $\mathrm{I}$ & $174(43.9)$ & $40(50.6)$ & $29(36.3)$ & $38(48.1)$ & $36(45.6)$ & $31(39.2)$ \\
\hline II & $104(26.3)$ & $19(24.1)$ & $23(28.8)$ & $18(22.8)$ & $21(26.6)$ & $23(29.1)$ \\
\hline III & $118(29.8)$ & $20(25.3)$ & $28(35.0)$ & $23(29.1)$ & $22(27.8)$ & $25(31.6)$ \\
\hline \multicolumn{7}{|c|}{ Pathology, $n(\%) \mathrm{e}^{\mathrm{e}}$} \\
\hline \multicolumn{7}{|c|}{ Adenocarcinoma } \\
\hline $\begin{array}{c}\text { Well- } \\
\text { differentiated }\end{array}$ & $212(53.5)$ & $41(51.9)$ & $36(45.0)$ & 47 (59.5) & $41(51.9)$ & 47 (59.5) \\
\hline $\begin{array}{c}\text { Moderately } \\
\text { differentiated }\end{array}$ & $159(40.2)$ & $33(418)$ & $34(42.5)$ & 25 (31.6) & $38(48.1)$ & $29(36.7)$ \\
\hline $\begin{array}{c}\text { Poorly } \\
\text { differentiated }\end{array}$ & $75(18.9)$ & $18(22.8)$ & $22(27.5)$ & $11(13.9)$ & $11(13.9)$ & $13(16.5)$ \\
\hline Signet ring cell & $40(10.1)$ & $14(17.7)$ & $9(11.3)$ & $9(11.4)$ & $5(6.3)$ & $3(3.8)$ \\
\hline Mucinous & $26(6.6)$ & $3(3.8)$ & $7(8.8)$ & $7(8.9)$ & $1(1.3)$ & $8(10.1)$ \\
\hline Papillary & $15(3.8)$ & $3(3.8)$ & $2(2.5)$ & $2(2.5)$ & $4(5.1)$ & $4(5.1)$ \\
\hline $\begin{array}{l}\text { Squamous cell } \\
\text { carcinoma, } n \\
(\%)\end{array}$ & $31(7.8)$ & $2(2.5)$ & $5(6.3)$ & $7(8.9)$ & $9(11.4)$ & $8(10.1)$ \\
\hline \multicolumn{7}{|c|}{ P53 expression, $n(\%)^{\mathrm{a}}$} \\
\hline $\begin{array}{l}\text { None } \\
\text { Faintly }\end{array}$ & $63(17.8)$ & $13(19.7)$ & $16(22.5)$ & $13(18.3)$ & $13(17.3)$ & $8(11.3)$ \\
\hline $\begin{array}{l}\text { expressed: }>0 \% \\
\&<10 \%\end{array}$ & $74(20.9)$ & $14(21.2)$ & $19(26.8)$ & $11(15.5)$ & $13(17.3)$ & $17(23.9)$ \\
\hline $\begin{array}{c}\text { Strongly } \\
\text { expressed: } \\
\geq 10 \% \&<50 \%\end{array}$ & $56(15.8)$ & 13 (19.7) & $14(19.7)$ & $12(16.9)$ & $10(13.3)$ & $7(9.9)$ \\
\hline $\begin{array}{c}\text { Overexpressed: } \\
\geq 50 \%\end{array}$ & $161(45.5)$ & $26(39.4)$ & $22(31.0)$ & $35(49.3)$ & $39(52.0)$ & $39(54.9)$ \\
\hline
\end{tabular}


Table 2. Cont.

\begin{tabular}{|c|c|c|c|c|c|c|}
\hline & Total $n=396$ & Q1 $n=79$ & Q2 $n=80$ & Q3 $n=79$ & Q4 $n=79$ & Q5 $n=79$ \\
\hline \multicolumn{7}{|c|}{ Vitamin D receptor expression, $n(\%)^{\text {a }}$} \\
\hline Q1, & $92(26.0)$ & $20(30.3)$ & $22(31.0)$ & $17(23.9)$ & $17(22.7)$ & $16(22.5)$ \\
\hline Q2, & $89(25.1)$ & $15(22.7)$ & $17(23.9)$ & $15(21.1)$ & $19(25.3)$ & $23(32.4)$ \\
\hline Q3, & $86(24.3)$ & $18(27.3)$ & $15(21.1)$ & $20(28.2)$ & $17(22.7)$ & $16(22.5)$ \\
\hline Q4, & $87(24.6)$ & $13(19.7)$ & $17(23.9)$ & $19(26.8)$ & $22(29.3)$ & $16(22.5)$ \\
\hline \multicolumn{7}{|c|}{ Ki67 expression, $n(\%)^{\text {a }}$} \\
\hline Q1, & $62(17.5)$ & $15(22.7)$ & $16(22.5)$ & $10(14.1)$ & $10(13.3)$ & $11(15.5)$ \\
\hline Q2, & $116(32.8)$ & $21(31.8)$ & $24(33.8)$ & $28(39.4)$ & $18(24.0)$ & $25(32.2)$ \\
\hline Q3, & $59(16.7)$ & 15 (22.7) & $8(11.3)$ & $11(15.5)$ & $15(20.0)$ & $10(14.1)$ \\
\hline Q4 & $117(33.1)$ & $15(22.7)$ & $23(32.4)$ & $22(31.0)$ & $32(42.7)$ & $25(35.2)$ \\
\hline $\begin{array}{c}\text { Adjuvant } \\
\text { chemotherapy, } \\
n(\%)^{\text {a }}\end{array}$ & $140(35.4)$ & $28(35.4)$ & $29(36.3)$ & $28(35.4)$ & 25 (31.6) & $30(38.0)$ \\
\hline
\end{tabular}

${ }^{a}$ Percentages may not sum to $100 \%$ because of rounding. ${ }^{b}$ IQR $=$ Interquartile range. ${ }^{c}$ Not measured in some patients. ${ }^{\mathrm{d}}$ Not measured in some patients. Calculated as weight in kilograms divided by height in meters squared. ${ }^{\mathrm{e}}$ Because many patients had multiple histopathologic components, histopathologic subgroups were not mutually exclusive of each other.

\subsection{Effects of Vitamin D Supplementation on Serum PD-L1 Levels}

The effects of vitamin D supplementation, as well as placebo, on serum PD-L1 levels were compared between pre (=after the surgery and just before starting supplement) and post (=1 year after starting supplement) supplementation in each quintile of the serum PD-L1 level (Figure 3). In the lowest quintile (Q1), vitamin D supplementation significantly up-regulated serum PD-L1 levels $(p=0.0008)$, with no significant change in the placebo group. On the other hand, in the highest quintile (Q5), vitamin D supplementation significantly down-regulated serum PD-L1 levels $(p=0.0001)$ despite no significant changes in the placebo group. On the other hand, vitamin D supplementation did not either upor down-regulate serum PD-L1 levels in the middle quintiles $(\mathrm{Q} 2, \mathrm{Q} 3$, and Q4) and in the total sample (all quintiles). 

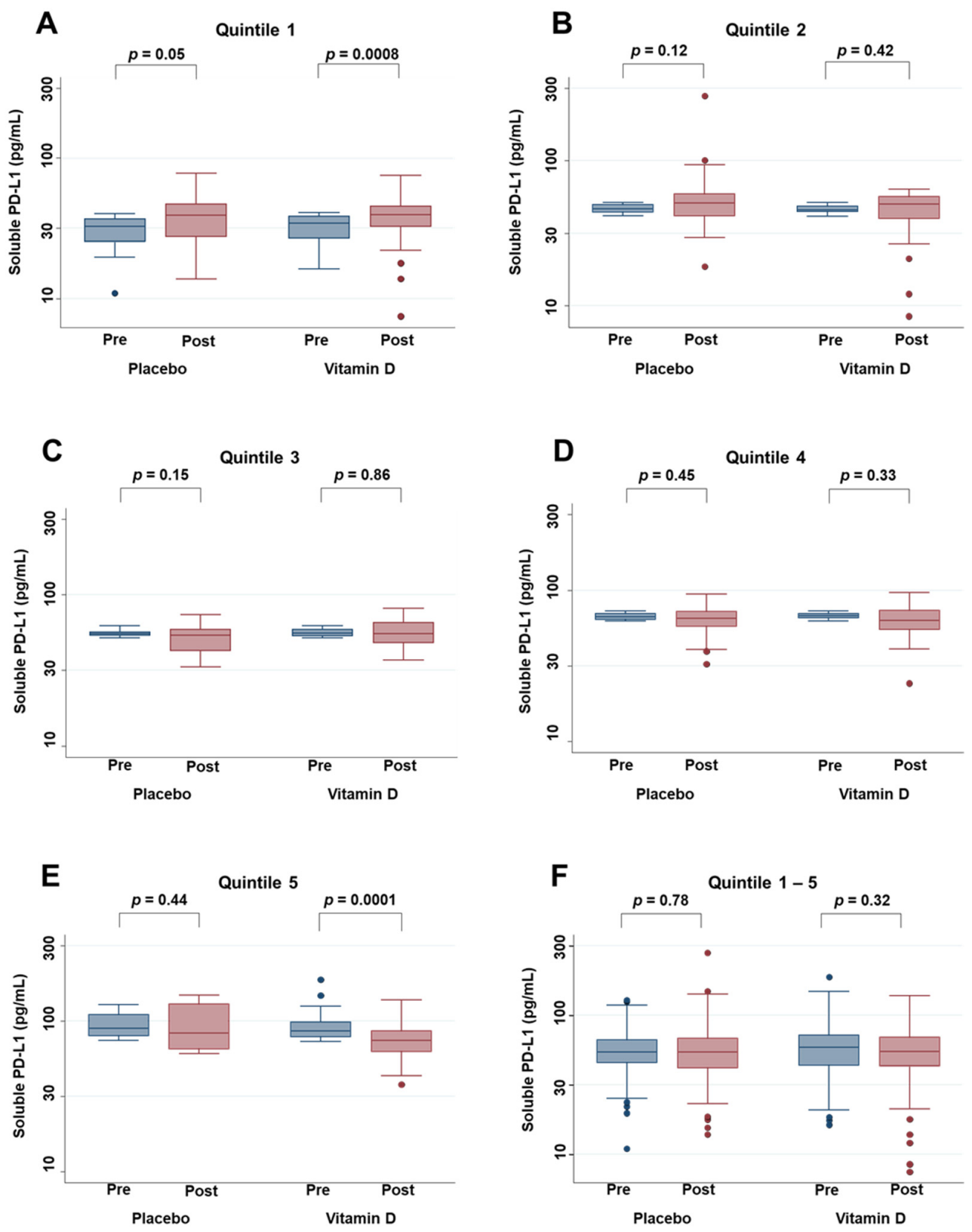

Figure 3. Box plot of changes in serum PD-L1 levels in the placebo group and the vitamin D group compared (A) for the subgroup of 1st quintile of PD-L1 levels (Quintile 1), (B) for the subgroup of 2nd quintile of PD-L1 levels (Quintile 2), (C) for the subgroup of 3rd quintile of PD-L1 levels (Quintile 3), (D) for subgroup of 4th quintile of PD-L1 levels (Quintile 4), (E) for subgroup of 5th quintile of PD-L1 levels (Quintile 5), (F) Sum of 1st-5th quintiles of PD-L1 levels. Pre = after the surgery and just before starting supplements; Post $=1$ year after starting supplementation. Changes between pre and post were evaluated with the Wilcoxon signed-rank test.

3.6. Effect of the Interaction between Vitamin D Supplementation and Serum PD-L1 Quintiles on Hazard Risk of Death

First, the effects of vitamin D supplementation on HRs of death were compared among quintiles of serum PD-L1 levels (Figure 4). A significant effect of vitamin D, compared with placebo, was observed in the highest quintile (Q5) of serum PD-L1 (HR 0.34; 95\% CI 0.12-0.92). On the other hand, significant effects of vitamin D on HRs of death were not observed in other quintiles, i.e., Q1 to Q4 and all quintiles excluding Q5 (Q1-Q4). There was a significant two-way interaction between the subgroup of Q5 and vitamin D supplementation ( $p$ for interaction $=0.04$ ), even on multivariate adjustment with (1) age, 
(2) sex, (3) body mass index, (4) cancer sites, i.e., esophageal, gastric, and small intestinal plus colorectal cancers, (5) stage, (6) adjuvant chemotherapy, and (7) p53 positivity ( $p$ for interaction $=0.02$ ).

A

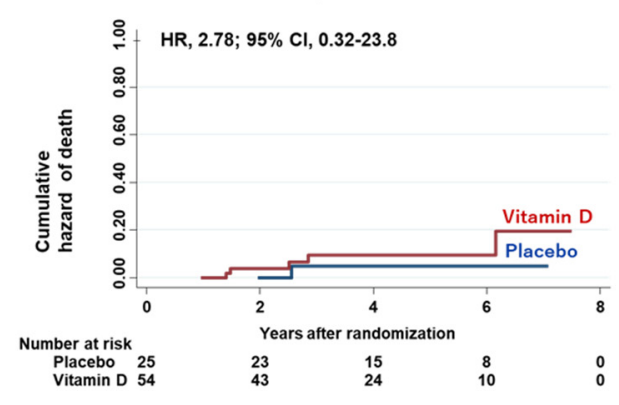

C

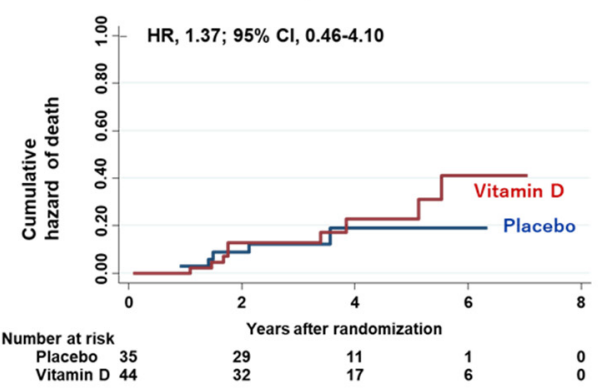

E

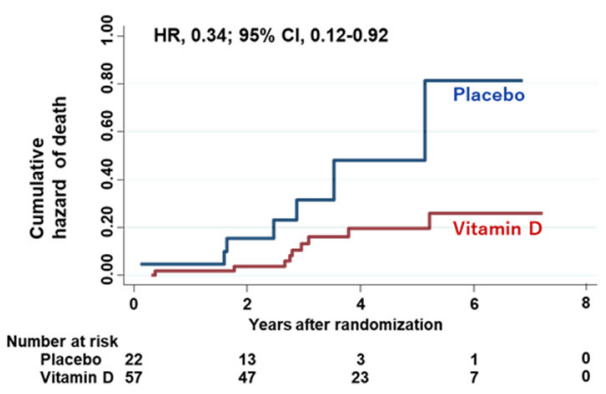

B

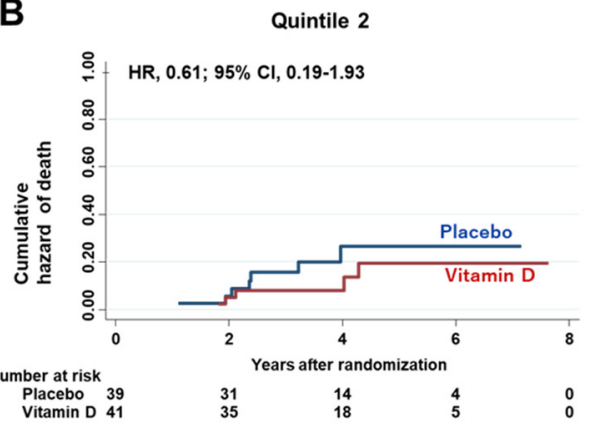

D

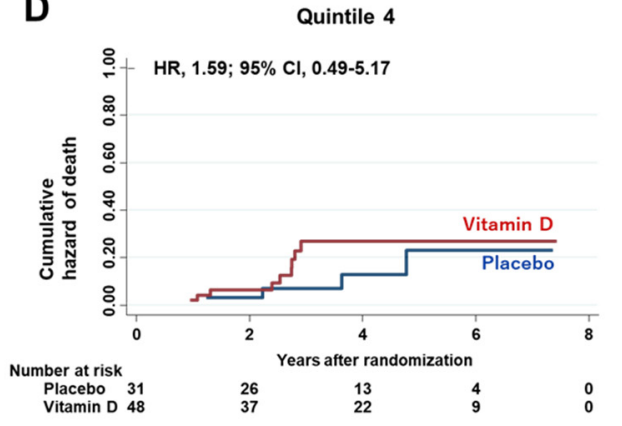

$\mathbf{F}$

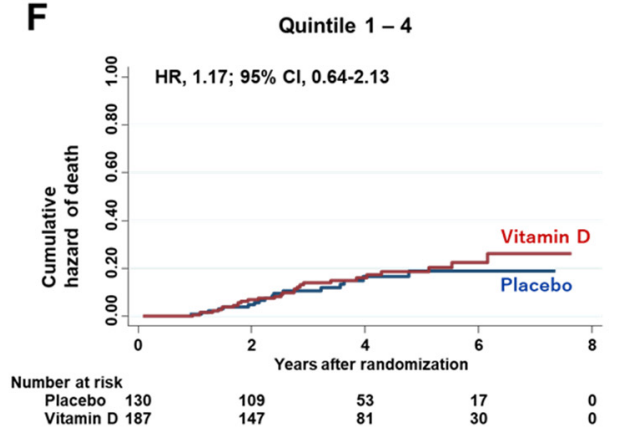

Figure 4. Cumulative hazard curves for death. Nelson-Aalen cumulative hazard curves (A) for death in the subgroup of 1st quintile of serum PD-L1 levels (Quintile 1), (B) for death in the subgroup of 2nd quintile of serum PD-L1 levels (Quintile 2), (C) for death in the subgroup of 3rd quintile of serum PD-L1 levels (Quintile 3), (D) for death in the subgroup of 4th quintile of serum PD-L1 levels (Quintile 4), (E) for death in the subgroup of 5th quintile of serum PD-L1 levels (Quintile 5), (F) for death in the subgroup of the sum of Quintile 1 to Quintile 4 serum PD-L1 levels. HR = Hazard ratio; $\mathrm{CI}=$ Confidence interval.

\subsection{Effect of the Interaction between Vitamin D Supplementation and Serum PD-L1 Quintiles on Hazard Risk of Relapse or Death}

Next, the effects of vitamin D supplementation on HRs of relapse or death were compared among quintiles of serum PD-L1 levels (Figure 5). Similarly, a significant effect of vitamin D, compared with placebo, was observed in the highest quintile (Q5) of serum PD-L1 (HR 0.37; 95\% CI 0.15-0.89). On the other hand, significant effects of vitamin D were not observed in other quintiles, i.e., Q1 to Q4 and all quintiles excluding Q5 (Q1-Q4). There was no significant two-way interaction between the subgroup of Q5 and vitamin D 
supplementation ( $p$ for interaction $=0.14$ ), but it became significant by adjustment with the same seven variables $(p$ for interaction $=0.04)$.

A

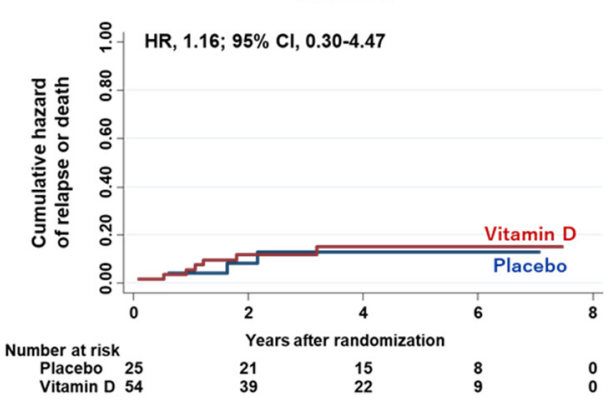

C

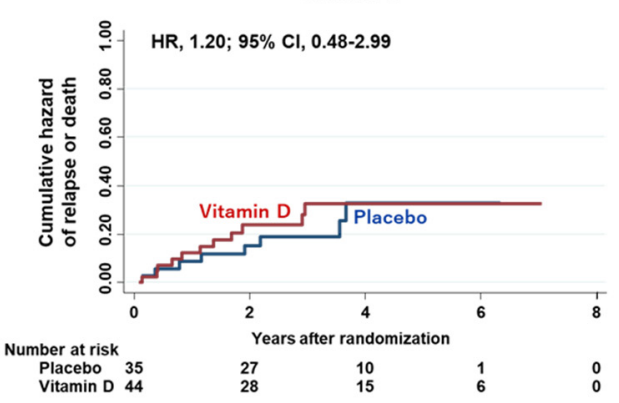

E

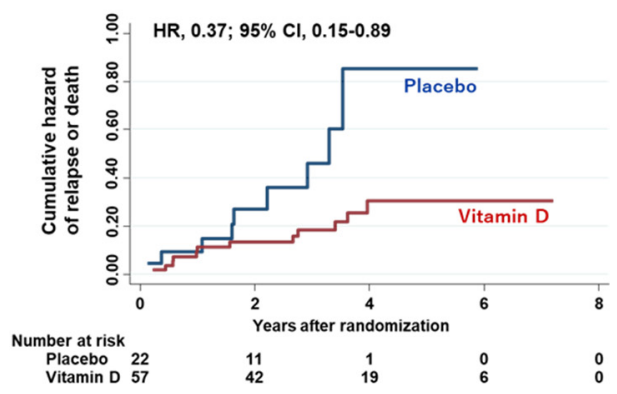

B

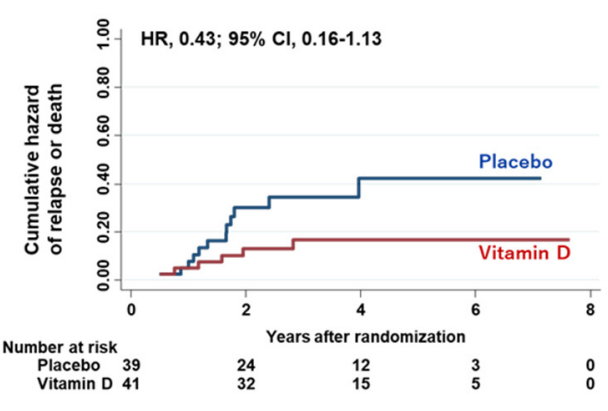

D

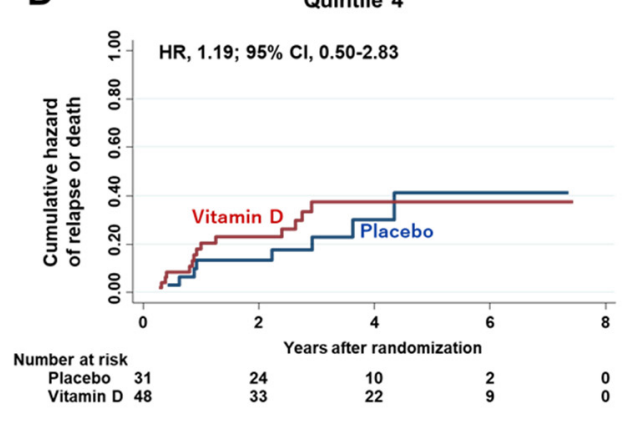

Figure 5. Cumulative hazard curves for relapse or death. Nelson-Aalen cumulative hazard curves (A) for relapse or death in the subgroup of 1st quintile of serum PD-L1 levels (Quintile 1), (B) for relapse or death in the subgroup of 2nd quintile of serum PD-L1 levels (Quintile 2), (C) for relapse or death in the subgroup of 3rd quintile of serum PD-L1 levels (Quintile 3), (D) for relapse or death in the subgroup of 4th quintile of serum PD-L1 levels (Quintile 4), (E) for relapse or death in the subgroup of 5th quintile of serum PD-L1 levels (Quintile 5), (F) for relapse or death in the subgroup of the sum of Quintile 1 to Quintile 4 of serum PD-L1 levels. HR = Hazard ratio; CI = Confidence interval.

Finally, the effects of vitamin D supplementation on the SHRs of relapse were compared in each quintile of serum PD-L1 levels. No significant effects of vitamin D, compared with placebo, were observed in all quintiles (Q1 HR 1.16, 95\% CI 0.30-4.40; Q2 HR 0.39, 95\% CI 0.13-1.13; Q3 HR 1.24, 95\% CI 0.48-3.22; Q4 HR 1.23, 95\% CI 0.46-3.29; and Q5 HR $0.45,95 \%$ CI $0.16-1.29)$. 


\section{Discussion}

In this clinical study, vitamin D supplementation up-regulated serum PD-L1 levels in the lowest quintile (Q1). This seems to be consistent with the results of experimental research that showed that vitamin D up-regulated expression of PD-L1 in epithelial and myeloid cells [13]. In contrast, vitamin D supplementation down-regulated serum PD-L1 levels in the highest quintile (Q5). Thus, vitamin D may have bimodal functions to increase serum PD-L1 when the serum PD-L1 levels are too low and to decrease serum PD-L1 when the serum PD-L1 levels are too high. However, further research regarding regulation of PD-L1 expression by vitamin D supplementation is needed.

Vitamin D supplementation, compared with placebo, significantly reduced the risk of total death, as well as relapse or death, to one-third in the highest quintile (Q5), but not in other quintiles, i.e., Q1-Q4, and did not change the risk of relapse. Because serum PD-L1 levels increased in an age-dependent manner in the present study and a previous report [19], multivariate adjustment including age was done and showed that they remained significant. In the present study, effects of the interaction between vitamin $\mathrm{D}$ and the highest quintile of serum PD-L1 were observed for the outcome of death rather than of relapse. Immune checkpoint inhibitors seem to improve overall survival rather than progression-free survival [20-22]. However, how PD-L1 is associated with death rather than relapse of patients has not yet been elucidated. Both the SUNSHINE [23] and AMATERASU [14] trials did not show significance in the primary results, although recent meta-analyses of RCTs suggested that vitamin D supplementation improved the survival of patients with cancer [24-27]. It has been hypothesized that vitamin D supplementation mainly reduces the risk of total death, at least in part by enhancing anti-cancer immunity and perhaps by keeping cancer tissue dormant by down-regulating serum PD-L1 levels.

This study has several limitations. First, exosomal PD-L1 was not measured in this study. However, not only exosomal PD-L1, but also total plasma PD-L1 was strongly associated with survival of patients with gastric cancer [10]. Second, serum PD-L1 levels were measured only after operation, but not before operation. However, postoperative rather than preoperative levels were reported to be associated with survival of patients with cancer [10]. Third, this study performed an exploratory analysis that was not pre-specified in the original protocol of the AMATERASU trial and must, therefore, be interpreted with caution. Fourth, subgroup analyses of quintiles may increase the probability of type I error due to multiple comparisons. A recent guideline for statistical reporting recommends replacing $p$ values with estimates of effects, such as $\mathrm{HR}$ and $95 \%$ CIs, when neither the protocol nor the statistical analysis plan has specified methods used to adjust for multiplicity [28]. Thus, $p$ values were avoided in the present study, except for calculating $p$ values for interaction and for changes in serum PD-L1 levels; instead, 95\% CIs were used to determine significance. Fifth, because the AMATERASU trial was conducted in Japan, the patients were Asian, most esophageal cancers were squamous cell carcinomas, the incidence of gastric cancer was still relatively high, and the optimal levels of total $25(\mathrm{OH}) \mathrm{D}$ and bioavailable $25(\mathrm{OH}) \mathrm{D}$ could be different from those in other population groups. Thus, the results of the present study are not necessarily generalizable to other populations. Sixth, the study population included patients with a mixture of cancers with biological and clinical differences.

\section{Conclusions}

Vitamin D supplementation, compared with placebo, may have bimodal functions to increase serum PD-L1 when the serum PD-L1 levels are too low and to decrease serum PD-L1 levels when the serum PD-L1 levels are too high. Vitamin D supplementation, compared with placebo, significantly reduced the risk of all-cause death, as well as relapse or death, to approximately one-third in the highest quintile (Q5), but not in other quintiles, i.e., Q1-Q4. Further studies are needed to explore the mechanisms of bimodal function of vitamin D in the secretion of serum PD-L1 in order to develop potential therapeutic opportunities by supplementation of vitamin $\mathrm{D}$. 
Author Contributions: Conceptualization, M.U.; methodology, M.M. and M.O.; formal analysis, M.M., and M.U.; resources, H.O. and Y.S.; data curation, T.A.; writing-original draft preparation, M.M. and M.U.; writing—review and editing, M.O., T.A., H.O. and Y.S.; funding acquisition, M.U. and M.M. All authors have read and agreed to the published version of the manuscript.

Funding: This research was funded by the Ministry of Education, Culture, Sports, Science, and Technology in the Japan-Supported Program for the Strategic Research Foundation at Private Universities, JSPS KAKENHI Grant Number 20H03537 (to M. Urashima), and the Jikei University Research Fund for Graduate Students (to M. Morita).

Institutional Review Board Statement: The study was conducted according to the guidelines of the Declaration of Helsinki and approved by the ethics committee of the International University of Health and Welfare Hospital (Otawara, Tochigi, Japan) (ethics approval code: 13-B-263), as well as the Jikei University School of Medicine (Nishi-shimbashi, Tokyo, Japan) (ethics approval code: 21-216 (6094)).

Informed Consent Statement: Informed consent was obtained from all subjects involved in the study.

Data Availability Statement: Publicly available datasets were analyzed in this study. This data can be found here: https:/ / upload.umin.ac.jp/cgibin/icdr/ctr_menu_form_reg.cgi?recptno=R000002412 (accessed on 5 April 2019).

Acknowledgments: The authors would like to thank Masumi Chida and Tomomi Ishikawa for making tissue microarrays and staining at the Department of Pathology, International University of Health and Welfare Hospital (Otawara, Japan); Yasuko Otsuki as a clinical research coordinator at the International University of Health and Welfare Hospital (Otawara, Japan); and Haruka Wada for data management and data monitoring at the Division of Molecular Epidemiology, Jikei University School of Medicine (Tokyo, Japan).

Conflicts of Interest: Makoto Morita is employed by Pfizer Japan Inc., but this work was performed independent of his work for that company. The authors declare no conflict of interest. The funders had no role in the design of the study; in the collection, analyses, or interpretation of data; in the writing of the manuscript, or in the decision to publish the results.

\section{References}

1. Boussiotis, V.A. Molecular and Biochemical Aspects of the PD-1 Checkpoint Pathway. N. Engl. J. Med. 2016, 375, 767-778. [CrossRef]

2. Gong, J.; Chehrazi-Raffle, A.; Reddi, S.; Salgia, R. Development of PD-1 and PD-L1 inhibitors as a form of cancer immunotherapy: A comprehensive review of registration trials and future considerations. J. Immunother. Cancer 2018, 6, 8. [CrossRef]

3. Zhu, X.; Lang, J. Soluble PD-1 and PD-L1: Predictive and prognostic significance in cancer. Oncotarget 2017, 8, 97671-97682. [CrossRef]

4. Romero, Y.; Wise, R.; Zolkiewska, A. Proteolytic processing of PD-L1 by ADAM proteases in breast cancer cells. Cancer Immunol. Immunother. 2020, 69, 43-55. [CrossRef]

5. Orme, J.J.; Jazieh, K.A.; Xie, T.; Harrington, S.; Liu, X.; Ball, M.; Madden, B.; Charlesworth, M.C.; Azam, T.; Lucien, F.; et al. ADAM10 and ADAM17 cleave PD-L1 to mediate PD-(L)1 inhibitor resistance. Oncolmmunology 2020, 9, 1744980. [CrossRef] [PubMed]

6. $\quad$ Frigola, X.; Inman, B.A.; Lohse, C.M.; Krco, C.J.; Cheville, J.C.; Thompson, R.H.; Leibovich, B.; Blute, M.L.; Dong, H.; Kwon, E.D. Identification of a Soluble Form of B7-H1 That Retains Immunosuppressive Activity and Is Associated with Aggressive Renal Cell Carcinoma. Clin. Cancer Res. 2011, 17, 1915-1923. [CrossRef] [PubMed]

7. Frigola, X.; Inman, B.A.; Krco, C.J.; Liu, X.; Harrington, S.M.; Bulur, P.A.; Dietz, A.B.; Dong, H.; Kwon, E.D. Soluble B7-H1: Differences in production between dendritic cells and T cells. Immunol. Lett. 2012, 142, 78-82. [CrossRef] [PubMed]

8. Okuyama, M.; Mezawa, H.; Kawai, T.; Urashima, M. Elevated Soluble PD-L1 in Pregnant Women's Serum Suppresses the Immune Reaction. Front Immunol. 2019, 10, 86. [CrossRef]

9. Li, X.; Zheng, Y.; Yue, F. Prognostic Value of Soluble Programmed Cell Death Ligand-1 (sPD-L1) in Various Cancers: A Metaanalysis. Target. Oncol. 2021, 16, 13-26. [CrossRef] [PubMed]

10. Li, G.; Wang, G.; Chi, F.; Jia, Y.; Wang, X.; Mu, Q.; Qin, K.; Zhu, X.; Pang, J.; Xu, B.; et al. Higher postoperative plasma EV PD-L1 predicts poor survival in patients with gastric cancer. J. Immunother. Cancer 2021, 9, e002218. [CrossRef]

11. Orme, J.J.; Enninga, E.A.L.; Lucien-Matteoni, F.; Dale, H.; Burgstaler, E.; Harrington, S.M.; Ball, M.K.; Mansfield, A.S.; Park, S.S.; Block, M.S.; et al. Therapeutic plasma exchange clears circulating soluble PD-L1 and PD-L1-positive extracellular vesicles. J. Immunother. Cancer 2020, 8, e001113. [CrossRef] [PubMed]

12. Feldman, D.; Krishnan, A.V.; Swami, S.; Giovannucci, E.; Feldman, B.J. The role of vitamin D in reducing cancer risk and progression. Nat. Rev. Cancer 2014, 14, 342-357. [CrossRef] 
13. Dimitrov, V.; Bouttier, M.; Boukhaled, G.; Salehi-Tarbar, R.; Avramescu, R.G.; Memari, B.; Hasaj, B.; Lukacs, G.L.; Krawczyk, C.M.; White, J.H. Hormonal vitamin D up-regulates tissue-specific PD-L1 and PD-L2 surface glycoprotein expression in humans but not mice. J. Biol. Chem. 2017, 292, 20657-20668. [CrossRef] [PubMed]

14. Urashima, M.; Ohdaira, H.; Akutsu, T.; Okada, S.; Yoshida, M.; Kitajima, M.; Suzuki, Y. Effect of Vitamin D Supplementation on Relapse-Free Survival Among Patients With Digestive Tract Cancers. JAMA 2019, 321, 1361-1369. [CrossRef] [PubMed]

15. Yonaga, H.; Okada, S.; Akutsu, T.; Ohdaira, H.; Suzuki, Y.; Urashima, M. Effect Modification of Vitamin D Supplementation by Histopathological Characteristics on Survival of Patients with Digestive Tract Cancer: Post Hoc Analysis of the AMATERASU Randomized Clinical Trial. Nutrition 2019, 11, 2547. [CrossRef] [PubMed]

16. Akutsu, T.; Okada, S.; Hirooka, S.; Ikegami, M.; Ohdaira, H.; Suzuki, Y.; Urashima, M. Effect of Vitamin D on Relapse-Free Survival in a Subgroup of Patients with p53 Protein-Positive Digestive Tract Cancer: A Post Hoc Analysis of the AMATERASU Trial. Cancer Epidemiol. Biomark. Prev. 2020, 29, 406-413. [CrossRef] [PubMed]

17. Urashima, M.; Okuyama, M.; Akutsu, T.; Ohdaira, H.; Kaji, M.; Suzuki, Y. Effect of Vitamin D Supplementation on Survival of Digestive Tract Cancer Patients with Low Bioavailable 25-Hydroxyvitamin D Levels: A Post Hoc Analysis of the AMATERASU Randomized Clinical Trial. Cancers 2020, 12, 347. [CrossRef] [PubMed]

18. Fine, J.P.; Gray, R.J. A Proportional Hazards Model for the Subdistribution of a Competing Risk. J. Am. Stat. Assoc. 1999, 94, 496-509. [CrossRef]

19. Chen, Y.; Wang, Q.; Shi, B.; Xu, P.; Hu, Z.; Bai, L.; Zhang, X. Development of a sandwich ELISA for evaluating soluble PD-L1 (CD274) in human sera of different ages as well as supernatants of PD-L1+ cell lines. Cytokine 2011, 56, 231-238. [CrossRef]

20. Hellmann, M.D.; Paz-Ares, L.; Caro, R.B.; Zurawski, B.; Kim, S.-W.; Costa, E.C.; Park, K.; Alexandru, A.; Lupinacci, L.; De la Mora, J.E.; et al. Nivolumab plus ipilimumab in advanced non-small-cell lung cancer. N. Engl. J. Med. 2019, 381, 2020-2031. [CrossRef]

21. Schmid, P.; Adams, S.; Rugo, H.S.; Schneeweiss, A.; Barrios, C.H.; Iwata, H.; Diéras, V.; Hegg, R.; Im, S.-A.; Wright, G.S.; et al. Atezolizumab and Nab-Paclitaxel in Advanced Triple-Negative Breast Cancer. N. Engl. J. Med. 2018, 379, 2108-2121. [CrossRef]

22. Eggermont, A.M.M.; Blank, C.U.; Mandalà, M.; Long, G.V.; Atkinson, V.; Dalle, S.; Haydon, A.; Lichinitser, M.; Khattak, A.; Carlino, M.S.; et al. Adjuvant Pembrolizumab versus Placebo in Resected Stage III Melanoma. N. Engl. J. Med. 2018, 378, 1789-1801. [CrossRef]

23. Ng, K.; Nimeiri, H.S.; McCleary, N.J.; Abrams, T.A.; Yurgelun, M.B.; Cleary, J.M.; Rubinson, D.A.; Schrag, D.; Miksad, R.; Bullock, A.J.; et al. Effect of High-Dose vs. Standard-Dose Vitamin D3 Supplementation on Progression-Free Survival Among Patients With Advanced or Metastatic Colorectal Cancer. JAMA 2019, 321, 1370-1379. [CrossRef] [PubMed]

24. Akutsu, T.; Kitamura, H.; Himeiwa, S.; Kitada, S.; Akasu, T.; Urashima, M. Vitamin D and Cancer Survival: Does Vitamin D Supplementation Improve the Survival of Patients with Cancer? Curr. Oncol. Rep. 2020, 22, 62. [CrossRef] [PubMed]

25. Vaughan-Shaw, P.G.; Buijs, L.F.; Blackmur, J.P.; Theodoratou, E.; Zgaga, L.; Din, F.V.N.; Farrington, S.M.; Dunlop, M.G. The effect of vitamin D supplementation on survival in patients with colorectal cancer: Systematic review and meta-analysis of randomized controlled trials. Br. J. Cancer 2020, 123, 1705-1712. [CrossRef] [PubMed]

26. Keum, N.; Lee, D.H.; Greenwood, D.C.; Manson, J.E.; Giovannucci, E. Vitamin D supplementation and total cancer incidence and mortality: A meta-analysis of randomized controlled trials. Ann. Oncol. 2019, 30, 733-743. [CrossRef]

27. Zhang, Y.; Fang, F.; Tang, J.; Jia, L.; Feng, Y.; Xu, P.; Faramand, A. Association between vitamin D supplementation and mortality: Systematic review and meta-analysis. BMJ 2019, 366, 14673. [CrossRef]

28. Harrington, D.; D'Agostino, R.B.; Gatsonis, C.; Hogan, J.W.; Hunter, D.J.; Normand, S.-L.T.; Drazen, J.M.; Hamel, M.B. New Guidelines for Statistical Reporting in the Journal. N. Engl. J. Med. 2019, 381, 285-286. [CrossRef] 